\title{
Le jeu de l'alternance dans la vie quotidienne des jeunes scolarisés à Dakar et à Ziguinchor (Sénégal)
}

Variation dans l'usage du français et du wolof

Language-switching in the Everyday Lives of School Children in Dakar and

Ziguinchor (Senegal): Variation on the Use of French and Wolof

Martine Dreyfus et Caroline Juillard

\section{(2) OpenEdition}

Journals

\section{Édition électronique}

URL : https://journals.openedition.org/etudesafricaines/115

DOI : 10.4000/etudesafricaines. 115

ISSN : 1777-5353

Éditeur

Éditions de l'EHESS

\section{Édition imprimée}

Date de publication : 1 janvier 2001

Pagination : 667-696

ISBN : 978-2-7132-1394-6

ISSN : 0008-0055

\section{Référence électronique}

Martine Dreyfus et Caroline Juillard, « Le jeu de l'alternance dans la vie quotidienne des jeunes scolarisés à Dakar et à Ziguinchor (Sénégal) », Cahiers d'études africaines [En ligne], 163-164 | 2001, mis en ligne le 21 novembre 2013, consulté le 12 mai 2021. URL : http://journals.openedition.org/ etudesafricaines/115; DOI : https://doi.org/10.4000/etudesafricaines.115 


\section{Martine Dreyfus \& Caroline Juillard}

\section{Le jeu de l'alternance dans la vie quotidienne des jeunes scolarisés à Dakar et à Ziguinchor (Sénégal)}

Variation dans l'usage du français et du wolof

\section{Du discours mixte comme manifestation du plurilinguisme urbain, au Sénégal}

Dakar et Ziguinchor ${ }^{1}$, la grande ville du Sud casamançais, sont des pôles migratoires drainant la migration rurale. Celle-ci entraîne d'importants brassages ethniques qui favorisent l'émergence d'un multilinguisme aux caractéristiques variables dans le temps et dans l'espace. Dans la capitale, comme à Ziguinchor, le multilinguisme régional s'est recomposé depuis le début $\mathrm{du} \mathrm{XX}^{\mathrm{e}}$ siècle et les mouvements des populations dans la ville le remodèlent sans cesse. À Dakar, le plurilinguisme régional qui s'organisait essentiellement autour du wolof, du lébou et, au niveau de l'ensemble de la région du Cap Vert, du sérère, s'est recomposé autour de trois pôles fédérateurs qui sont le wolof, langue dominante du milieu, le français et les langues des migrants: essentiellement le peul, le sérère (régions du Sine et du Saloum), le manding, le diola (Dreyfus 1995). À Ziguinchor le multilinguisme est beaucoup plus équilibré, autour de trois langues majoritairement utilisées : diola, wolof et manding, et d'une diversité d'autres langues régionales également présentes en ville : peul, mancagne, manjak, créole portugais, balant, etc. L'expansion du wolof y est récente, son usage progresse du centre ville, commercial et administratif, à la périphérie, au mode de vie proche de celui du monde rural avoisinant; cette langue tend à prendre la position dominante dans la nouvelle configuration multilingue (Juillard 1995).

Le multilinguisme et ses avatars formels autant que symboliques témoignent d'identités, personnelles autant que collectives, composées, résultant de contacts sur des périodes variables, dans l'espace familial, la concession,

1. 1500000 habitants pour Dakar en 2000 et 124000 habitants en 1988 pour Ziguinchor. 
le village, la ville, la région. Dans le cadre d'une situation postcoloniale, commune à différents États africains, le pluriethnisme se restructure en ville, entre tradition et nouveaux syncrétismes, et les identités de référence sont plus mouvantes et enchâssées : on peut mobiliser, tour à tour ou quasisimultanément, diverses identités, qu'elles soient nationale, régionale, ethnique et/ou villageoise, urbaine/de quartier, etc. La distinction qui s'accroît entre le monde rural et le monde urbain a pour effet de renforcer les modèles d'identification à l'un ou à l'autre. Les jeunes urbains sont les moteurs de ces différenciations en cours. Dans le cas de l'urbanisation en Afrique, différents auteurs (Parkin 1977 ; Manessy 1995 ; Myers Scotton 1993 ; Wald 1994 ; Thiam 1994) ont mis en évidence les modifications de formes et de fonctions linguistiques, concomitantes de ces évolutions. La coïncidence, dans le temps, des descriptions sociolinguistiques de Dakar et de Ziguinchor, de même que la concordance de leurs objectifs et de leurs méthodologies, a permis d'ébaucher une comparaison, sous cet aspect, entre ces deux villes liées par un passé historique commun, des liens administratifs et commerciaux, des liens migratoires surtout.

Les enquêtes ont montré que la communication en ville, au Sénégal comme en Afrique en général, est souvent bilingue, voire plurilingue, que ce soit lors d'échanges à caractère informel et ouvert en famille ou entre pairs, par exemple, ou encore dans des situations plus normalisées, ritualisées et codifiées telles des transactions commerciales, des réunions de travail, des séances d'enseignement (Dreyfus 1995; Juillard 1995 ; Myers Scotton 1993 ; Swigart 1992). Cette problématique de la «rencontre » des langues, d' «entre les langues », peut être posée à l'aide des notions de centre et de périphérie (Juillard \& Wald 1994) : Dakar fonctionnant comme un modèle de la diffusion du wolof (langue nationale, principale langue véhiculaire du Sénégal et principale langue parlée dans la région de Dakar) et du français (langue officielle) face à la périphérie des villes secondaires, telle Ziguinchor, où s'enracine la diversité des langues locales. Ce rapport implique aussi la vernacularisation du wolof, au centre, et sa véhicularisation à la périphérie. Le modèle centre/périphérie fonctionne également dans la capitale, le français occupant les espaces centraux : quartiers administratifs, quartiers du grand commerce, sur le Plateau et dans ses environs immédiats; le wolof au centre de la ville également mais se diffusant de plus en plus vers la périphérie alors que diminue l'usage du français, et que l'on note la présence plus sensible de certaines langues locales aux marges de l'agglomération dans des quartiers de « déguerpis » et des quartiers d'habitation spontanée dans les zones interstitielles de la ville ou à la périphérie ${ }^{2}$.

2. Les migrants arrivés à Dakar, sans travail, occupent des quartiers « d'urbanisation spontanée », bidonvilles centraux, proches des zones d'embauche, et peu à peu se crée une répartition sociale de l'espace urbain, entre le Plateau, ancienne ville coloniale, et les cités de moyen standing destinées à la bourgeoisie moderne sénégalaise, les quartiers populaires de la Médina, et de Grand Dakar, lieux d'accueil des migrants et les «baraques-villes» des zones d'habitation spontanée, 
À Dakar, le wolof et le français sont souvent présentés par les locuteurs comme les langues d'intégration urbaine, les langues de la ville, et le « code mixte wolof-français » ou le «wolof urbain » ${ }^{3}$, wolof très mêlé ou alterné d'éléments empruntés au français et aux autres langues africaines, est, d'après les descriptions amorcées, une variété partagée par un nombre de plus en plus grand de locuteurs y compris par ceux qui n'ont pas été scolarisés (Daff 1995 ; Thiam 1998 ; Ndao 1996 ; Dreyfus 1999 ; Swigart 1992). Nous utiliserons désormais dans le cadre de cet article le terme de discours mixte pour prendre en compte l'ensemble des usages mélangés rencontrés à Dakar et à Ziguinchor.

L'étude des répertoires linguistiques contrastés des jeunes et des adultes dakarois révèle une grande diffusion du discours mixte wolof-français auprès de la plupart des locuteurs. Une première approche de la fonctionnalisation de ce code à Dakar peut être faite à partir de données recueillies lors d'une recherche effectuée auprès d'enfants et d'adolescents par questionnaires, entretiens et observations sur les usages linguistiques dans la famille, entre pairs et dans le quartier (Dreyfus 1995). Les langues sont dans ce type d'approche méthodologique envisagées comme des entités linguistiques distinctes, déjà constituées, qui peuvent être pour les locuteurs des objets de choix en fonction de différentes situations de communication. Les langues déclarées les plus souvent associées, mélangées et/ou alternées dans les interactions familiales, quelle que soit la langue d'origine des locuteurs, sont le wolof et le français. La fréquence d'emploi la plus importante se trouve dans la fratrie $(39,7 \%$ des locuteurs déclarent utiliser le wolof en alternance avec le français avec leurs frères et sœurs) puis dans les interactions enfant-père $(31,4 \%$ des enfants interrogés déclarent utiliser l'alternance wolof-français lorsqu'ils s'adressent à leur père). Cet usage alterné ou mêlé est nettement moins fréquent dans la communication entre la mère et l'enfant : 16,7 \% lorsque l'enfant s'adresse à sa mère. Si l'on considère les usages, on peut relever les cas où le wolof est langue commune des deux parents ; ceux-ci servent en quelque sorte de groupe de référence, puisqu'ils constituent non seulement le groupe numériquement dominant, mais également celui dont la langue est véhiculaire et en voie de vernacularisation dans les grands centres urbains du nord du pays. Les langues utilisées entre les parents sont, pour l'essentiel, le wolof, langue déclarée employée seule,

régulièrement « déguerpis » pas les autorités urbaines à la périphérie de la ville, et régulièrement réinvestis par de nouveaux occupants.

3. Ces dénominations sont celles qui sont les plus couramment utilisées par les chercheurs ayant travaillé dans ce domaine au Sénégal. Le parler mixte et/ou alterné wolof/français est également appelé « wolof urbain » par SwIGART (1990, 1992), « code mixte wolof français » par Dreyfus (1995) et Thiam (1994), « francolof» par Diouf (1991) ou encore «Colonial dialogue : Language Mixing in Dakar» par ReINSch (1994). Ces différentes appellations témoignent, entre autres, de la difficulté à décrire cette variété à partir de pratiques discursives extrêmement variées, fortement marquées par les stratégies individuelles et les différents enjeux communicationnels des locuteurs. 
sans alterner avec le français ni avec d'autres langues dans plus de $80 \%$ des cas mais l'alternance domine dans la fratrie avec un usage étendu du wolof associé au français : 55,3\% des enfants déclarent alterner ou mélanger les deux langues.

Le wolof urbain est déclaré utilisé « très souvent » par les jeunes, quelle que soit leur langue d'origine, et majoritairement dans les interactions qui ont pour cadre les échanges entre pairs et les échanges quotidiens dans les différents quartiers (relations de voisinage, transactions commerciales) : les pourcentages varient de plus de $98 \%$ des locuteurs à $79 \%$ (ce dernier pourcentage concerne des quartiers où existent certains regroupements de communautés d'origine peul, manding, diola ou serer; globalement, à Dakar, la majorité des quartiers est très hétérogène du point de vue du peuplement). Ces usages mélangés traversent différents espaces sociaux, à Dakar, et tendent également à remplir des fonctions identitaires dans différents groupes de la société urbaine (Swigart 1990, Thiam 1998).

À Ziguinchor, également, la pratique du mélange de langues est fréquente et habituelle chez beaucoup de locuteurs et pénètre l'espace des langues emblématiques du groupe d'appartenance. C'est la part plus ou moins importante du wolof, voire du français, dans ce discours mixte qui crée la différence entre les usages des différentes générations en présence, des hommes et des femmes, des scolarisés et des non scolarisés. Cependant, l'affirmation identitaire par l'usage revendiqué (sinon constant) de la langue de groupe reste encore possible pour la très grande majorité des citadins : si la concentration ethnique par quartiers ou par zones de regroupement est encore sensible en ville, c'est au centre ville que l'usage de la langue de groupe se maintient le moins. Les enquêtes réalisées par Caroline Juillard à Ziguinchor ont fait ressortir tant le plurilinguisme des enfants en famille, que l'effet emblématique de la langue de groupe : si, en famille, la pratique d'usages bi- ou plurilingues est déclarée par la grande majorité des jeunes interrogés, le wolof vient en deuxième position d'usage déclaré pour la majorité des jeunes interrogés, après la langue de groupe.

On peut voir cette situation, à Dakar principalement, mais également à Ziguinchor, comme le résultat d'une double évolution : le français investit des espaces sociaux jusque là réservés aux langues africaines et celles-ci pénètrent également les espaces sociaux traditionnellement dévolus au français (Daff 1995 ; Ndao 1996 ; Thiam 1998). Les observations faites ${ }^{4}$ confirment leur présence dans l'administration et dans de nombreux autres espaces

4. Toutes les observations réalisées ces quinze dernières années, par différents chercheurs, concordent. Le commentaire suivant illustre bien cette évolution : « À partir de 1980, le français normé n'est utilisé que dans des situations formelles d'où la convivialité est exclue [...] » (Daff 1995). Jusqu'à une date récente, seul le français était utilisé dans toutes les interactions dans l'enceinte de l'université. Aujourd'hui le wolof est aussi fréquent que le français dans les couloirs et bureaux de l'administration locale. 
institutionnels où normalement devrait apparaître la langue officielle. L'appropriation du français par les locuteurs au Sénégal est à la fois liée à une pratique fonctionnelle, à l'utilisation «d'une ressource spécialisée qu'est le français institutionnel» (Wald 1994) et l'utilisation du français avec le wolof, à Dakar, dans le discours mixte, traduit une appropriation « vernaculaire ». Dans ce qui semble être en voie de devenir une nouvelle variété linguistique, le français, associé au wolof, remplit une fonction identitaire et le locuteur se situe alors dans un contexte «local» d'usager de cette langue. La langue française, qui fonctionnait sur un mode diglossique, pour un nombre très limité de personnes, s'est banalisée progressivement dans la capitale, où son appropriation s'opère actuellement de plus en plus hors du système scolaire. Elle s'est lentement popularisée, par le biais de formes moins surveillées parlées par des personnes peu scolarisées, étrangers ou autochtones, dans les situations informelles de la vie quotidienne. Le discours unilingue en français reste réservé à des situations très formelles ; il en est de même pour le discours dans la langue de groupe. Le discours bilingue tend à devenir la norme de communication pour certains jeunes scolarisés. C'est également au travers de nombreux emprunts, consolidés ou spontanés, que se traduit l'importance de cette langue dans le répertoire.

Face à un unilinguisme possible, en langue de groupe, en wolof ou en français, dans certaines situations et certains rapports d'interlocution, le bilinguisme à dimension variable est un recours fréquent qui permet un positionnement interpersonnel souple; l'usage d'éléments de wolof et de français est incontournable pour les jeunes citadins dans ce nouvel équilibrage des langues en milieu urbain, tant à Dakar qu'à Ziguinchor. N. Thiam (1998) souligne que «les pratiques langagières en milieu urbain font appel constant au français et au wolof, langues glottophages pour les autres langues, mais pas entre elles ». Le mélange des langues est une alternative au passage de l'une à l'autre. Quelles sont les modalités, la fonctionnalité et la variabilité de ce mélange ? Nos illustrations de ces discours mixtes ou mélanges tels qu'ils peuvent être saisis dans des conversations ordinaires en sont un témoignage.

Ce sont les enregistrements d'interactions, in situ, qui manifestent le mieux la dynamique de la parole dans ses usages bilingues. Le mélange linguistique observé au sein des interactions est un élément émergent porteur d'un symbolisme co-construit par les agents, et le référent de leur univers sociolinguistique, propre autant que collectif, constamment en mouvement et recréé. Il faut noter également l'extrême variabilité des données sur lesquelles nous travaillons, extrêmement labiles et effets de multiples causes individuelles, collectives, sociales, linguistiques, discursives. On peut tenter cependant de trouver des éléments de convergence dans les pratiques bilingues propres aux jeunes scolarisés, tant à Dakar qu'à Ziguinchor. Par ailleurs, les différences constatées font-elles ressortir des types de mélange distincts, des récurrences, des particularismes propres à l'une ou l'autre des situations urbaines ? Comment, dans ces deux villes, le wolof et le français s'interpénètrent-ils ou pénètrent-ils « à l'intérieur» des langues de groupes ? 


\section{Cadre interprétatif du discours mixte}

La variété de langue qui est utilisée et présentée à travers les extraits est caractéristique des pratiques langagières des personnes qui ont été scolarisées et se diffuse de plus en plus chez les jeunes et les adultes non scolarisés. L'ensemble des corpus constitués, d'où sont tirés les fragments présentés dans le cadre de cet article, concerne essentiellement, pour Dakar, des enfants, des adolescents et des adultes lettrés ; pour Ziguinchor, il ne concerne que des jeunes scolarisés.

L'approche que nous faisons du contact de langues privilégie l'étude de la pluriconstruction du sens et des agencements identitaires. Ces pratiques mixtes permettent à un individu de créer à travers des choix de langues, d'alternances ou de mélanges, de nouvelles formes d'usage, et donc, de nouvelles possibilités d'expressions identitaires. L'analyse de ces pratiques mixtes s'inscrit dans le cadre général des approches développées à la suite des travaux de J. J. Gumperz et de C. Myers Scotton notamment, qui mettent en avant l'activité langagière du sujet et des effets obtenus à travers l'alternance ou le mélange de langues. Myers Scotton (1993: VII, 57 s.) note comment le recours au code switching (CS) permet de négocier les identités sociales dans l'interaction et s'avère être une ressource pragmatique : «Codeswitching is used to convey intentionnel (i.e. non-code-based) meaning of a socio-pragmatic nature. » Rappelons brièvement le postulat de base de cette théorie : le choix d'un cadre linguistique reflète les connaissances partagées des interlocuteurs au sujet d'un ensemble de droits et d'obligations sociaux et intra-individuels. Tout locuteur dans une situation donnée peut choisir non seulement le code non marqué par rapport à cette situation, qui correspond aux droits et aux obligations attendues entre ces deux partenaires et lorsqu'il désire changer l'équilibre de ces droits et obligations, il peut changer de code vers une variété marquée pour l'acte de parole en question. C. Myers Scotton (1993 : 114) distingue plusieurs alternatives : «Speakers engage in CS in one of these four related types: (1) CS as a sequence of unmarked choices ; (2) CS itself as the unmarked choice ; (3) CS as a marked choice ; (4) CS as an exploratory choice. » Elle se réfère notamment au principe de «co-operative principle» de P. Grice: «Choose the form of your conversation contribution such that it indexes the set of rights and obligations which you wish to be in force between speaker and adressee for the current exchange. » Par exemple, dans un échange avec un subordonné initié en wolof (code non marqué) sur le lieu de travail, un locuteur sénégalais peut affirmer son autorité en passant du wolof au français, pour marquer son pouvoir sur la prise de décision ou imposer un ordre ou au contraire mettre l'accent sur sa solidarité avec l'interlocuteur en continuant en wolof; le même locuteur pourra utiliser un code mixte wolof-françaissérère, s'il est d'origine sérère, au cours d'une interaction avec son fils et passer au français (code marqué). Le discours mixte trouve sa place dans ce modèle car l'alternance peut en elle-même constituer un choix non marqué : les deux ensembles de droits et d'obligations s'appliquent alors 
simultanément et plusieurs aspects de l'identité du locuteur plurilingue sont saillants en même temps.

J. J. Gumperz (1989: 60) avance que les locuteurs se fondent sur la compréhension abstraite des normes situationnelles et interactionnelles «pour communiquer une information métaphorique sur la façon dont ils veulent que leurs paroles soient comprises » et donc pour construire du sens. D'un point de vue sémantique et discursif, il reconnaît six fonctions susceptibles de produire un sens supplémentaire dans l'alternance codique conversationnelle et donc de marquer les stratégies discursives utilisées par les locuteurs. La plupart de ces fonctions sont déjà conventionnellement et formellement marquées dans un discours monolingue (par exemple, les fonctions suivantes : citation; désignation de l'interlocuteur; interjection ; réitération). Mais les deux dernières fonctions : la qualification-modalisation $\mathrm{du}$ message et la fonction de personnalisation vs objectivation, indiquent la façon dont les locuteurs situent leurs propos et se situent par rapport à eux et apparaissent plus spécifiques des possibilités de stratégies discursives offertes par le discours mixte (Nicolaï 1986).

Notre étude emprunte également aux outils développés par l'analyse de discours en France et aux recherches conversationnelles ou interactionnelles. Dans le prolongement des travaux de Gumperz sur les fonctions du CS et dans les approches les plus récentes des discours mixtes, le point de vue discursif, énonciatif et conversationnel est nettement privilégié dans l'étude sémantique de l'alternance de langues ; celle-ci est souvent considérée comme marqueur du changement des genres discursifs et comme révélateur de l'hétérogénéité énonciative du locuteur (en référence au dialogisme de Bakhtine ou à l'hétérogénéité du dire d'Authier-Revuz) ${ }^{5}$.

Il est également important de tenir compte, dans l'analyse explicative des discours mixtes, de la dimension interactionniste des situations de contact pour étudier comment les interlocuteurs collaborent afin de coconstruire du sens en utilisant toutes les ressources de leur répertoire : « La rencontre des langues en contact dans l'interaction suscite des phénomènes qui ne sont totalement réductibles ni aux propriétés des systèmes en présence, ni à la compétence des interlocuteurs, ni aux déterminations sociales extra-linguistiques. Dans cette optique, l'évènement langagier est donc appréhendé en tant qu'il constitue le produit d'une construction collective des acteurs sociaux »(Gumperz 1989: 59) ${ }^{6}$.

5. Cf., pour exemple, le travail d'A. Bensalah (1998) sur les marqueurs de l'énonciation ou énonciatifs, en tant que trace de la relation du sujet énonciateur à ses interlocuteurs, dans des récits de vie bilingues algérien/français.

6. On peut citer, en écho, J. Boutet (1994: 26) : «L'analyse du langage socialement situé implique une réflexion sur la production de sens par les acteurs sociaux car le linguiste se trouve confronté non seulement à la production des énoncés mais aussi à leur réception et à leur compréhension sociales particulières. S'attacher à la production et à la compréhension des énoncés c'est envisager l'activité langagière comme une activité avant tout signifiante et c'est mettre la question du sens au cœur de la réflexion : les langues sont alors conçues comme productrices de sens et pas seulement comme des systèmes formels. » 
Le recours à plusieurs langues ou variétés apparaît ainsi à la fois comme un moyen de représenter et de symboliser des identités sociales multiples dans un univers plurilingue, et comme une ressource supplémentaire dans la production de sens ou dans l'utilisation de stratégies discursives et interactionnelles appropriées.

\section{Formes du discours mixte}

Le mélange ou l'alternance de langues dans ce que nous nommons discours mixte en référence à notre terrain peut prendre plusieurs formes tout comme il peut résulter de plusieurs processus. Dans certains cas, ils peuvent caractériser un état transitoire qui marque un changement ( language shift») linguistique et social dans lequel une communauté passe d'une situation de plurilinguisme à une situation de monolinguisme, et/ou une inversion de dominance sociolinguistique : abandon progressif de la ou des langues de la communauté au profit d'une autre langue.

En Afrique, les situations plurilingues existent depuis longtemps, et les changements linguistiques qui ont probablement eu lieu dans le passé (Doneux 1977) (adoption et abandon de langues ou émergence de lingua franca) ne semblent pas avoir amené une évolution nette vers un monolinguisme. Le cas de Ziguinchor est de ce point de vue exemplaire, comme la présence à Dakar des langues des communautés peul, toucouleur, diola ou sérère à côté du wolof et du français. C. Myers Scotton (1993 : chap. I), qui a travaillé plusieurs décennies au Kenya et au Zimbabwe, partage ce point de vue : «[...] Such conversations are not mainly a transitionnal stage in a language shift from dominance in one language to another. It is true that many immigrants who are in the process of language shift do engage in code switching, but this form of conversation is also part of daily lives of many "stable" bilingual populations as well. "

Nous désignons sous le terme discours mixte l'alternance, le mélange ou le changement de langues ou de variétés linguistiques dans un discours ou dans une conversation; nous utiliserons par la suite le simple terme d'alternance pour désigner cet ensemble de phénomènes et le terme discours mixte pour nommer cette variété linguistique.

— L'alternance peut être «interphrastique » :

Exemple 1:

Échanges à visée explicative ; un garçon : 20 ans, explique à son frère la résolution d'une équation mathématique :

Donc on peut conclure ++ alors $\boldsymbol{x}+\boldsymbol{y}=++$ xoolal nii lañuy def : $\mathrm{f}(\mathrm{z})$ nee nañu lii la ++ Alors on essaye de mettre ceci sous cette forme en remplaçant chaque fois $z$ par $x$ et $y++$ On peut donc déduire +++ 
Donc on peut conclure +++ alors $\mathrm{X}+\mathrm{Y}=+++$. Regarde c'est comme ça qu'on fait [ $\mathrm{f} \mathrm{de} \mathrm{z}$ ] on dit que c'est ça. Alors on essaie de mettre ceci sous cette forme en remplaçant chaque fois $\mathrm{Z}$ par $\mathrm{X}$ et $\mathrm{Y}$ on peut donc déduire +++ .

— ou « intraphrastique » :

Exemple 2 :

Échanges lors d'un repas : une fille, 18 ans, parle avec ses frères du thème d'une chanson, Le déserteur, de Léo Ferré :

Nee moom du + elle n'est pas + homme + du wér pour la guerre. Na ne mënul ñów mu ne président bi je t'avertis de toutes les façons + ak nu mu mënti mel je vais m'évader tey pour langue + daw guerre.

Il dit (que) lui n'est pas [elle n'est pas homme] bon [pour la guerre] il dit qu'il ne peut pas venir il dit [président] bi (class.) [je t'avertis de toutes les façons-] et comme il ne peut pas [je vais m'évader] aujourd'hui pour [quoi] fuir [(la) guerre].

Dans le cas d'une alternance intraphrastique, celle-ci peut intervenir entre deux syngtames : je vais m'évader (S. V) tey (S. P)

ou à l'intérieur d'un syntagme : pour lan + daw guerre

ou au niveau morphématique, dans un même constituant.

Exemple 3 :

Échanges pendant une partie de jeux de billes :

Pouss- $a l^{7}$ duma xam lu may jouer

pousse je ne sais pas ce que je joue

pouss + al : rad. + impératif $2^{\mathrm{e}}$ personne sing.

lu may jouer : lu interrogatif indir. + ma pronom personnel première personne + y marque de l'imperfectif présent + jouer

— ou «extraphrastique » d'un tour de parole à un autre :

Exemple $4^{8}$ :

Les joueurs jouent et parlent à la fois. Le rythme est très rapide :

111 sayken galab

parle vite!

112 partie jouée

113 ah oui, quand c'est lent

7. «Poussal »: cette alternance au niveau d'un léxème est à replacer dans le processus de l'emprunt (ici emprunt en cours d'intégration).

8. Échanges pendant une partie de cartes à Ziguinchor. Les langues alternées sont : le diola (caractères simples), le français (caractères simples soulignés), le manding (caractères gras soulignés). 
114 fulempafu fia foofu

ta barbe (= le roi de cœur), voilà

115 amãykolya !... sõnoleemu !

ce n'est pas clair ! [...] c'est un fraudeur

116 kolyatale !

ah! c'est clair (= il n'y a pas de vol)

\section{7 amã kolya !}

ce n'est pas clair!

118 he waw?

$$
\text { et qu'est-ce qu'il y a ? }
$$

\section{9 sãyoleemu !} c'est un fraudeur!

120 ha nata...

Ah bon, j'ai pris...

121 e ! ulal kasãykenku mãndinay kute

ah nous sommes en train de nous exprimer en manding ici

122 e ! mo jajak (rires)

ah ! c'est positif

Les éléments appartenant aux deux variétés linguistiques dans une même phrase sont généralement liés d'un point de vue prosodique. Cette définition est comparable en certains points à celle proposée par C. Myers Scotton (1993 : 4) qui y inclut la notion de langue de base (notion que nous utilisons également mais que nous ne discuterons pas formellement dans le cadre de cet article) : «Codeswitching is the selection by the bilinguals or multilinguals of forms from an embedded language (or languages) in utterances of a matrix language during the same conversation. [.... Stretches of codeswitching material may be intersentential or intrasentential [...], intrasentential switches occur within the same sentence, from single morpheme to clause level. »

C'est certes une définition très générale, qui n'est pas sans ambiguïté notamment dans la délimitation des variétés, puisqu'elle renvoie à la fois aux langues en elles-mêmes, aux formes véhiculaires et vernaculaires de ces langues, aux registres, etc., mais elle rend bien compte de l'ensemble des discours mixtes rencontrés en Afrique dans des sociétés plurilingues.

\section{Le discours mixte à Dakar}

Nous avons sélectionné deux illustrations ${ }^{9}$ des formes que peut prendre le discours mixte à Dakar. Ce sont des usages très fréquemment observés et attestés par les corpus produits dans le cadre de différentes recherches (Daff 1995 ; Dreyfus 1999 ; Dumont 1973 ; Ndao 1996 ; Thiam 1998 ; Swigart 1990)

9. Ces extraits ont été présentés ailleurs (Dreyfus 1999), mais non analysés. 
et reconnus par une catégorie de locuteurs : les jeunes, comme emblématiques d'une identité urbaine et/ou d'une identité de groupe (Swigart 1990). S'ils comportent essentiellement une alternance de wolof et de français, et s'ils apparaissent très répandus à Dakar, ils ne sont cependant pas exclusifs d'autres formes de discours mixtes, également présents en ville, où peuvent alterner wolof, français et d'autres langues locales.

Le premier extrait de corpus comporte des conduites discursives à visée explicative et argumentative ; il illustre en quelque sorte l'aspect fonctionnel du discours mixte wolof/français à Dakar.

Les conduites discursives se définissent non seulement par l'emploi de formes linguistiques ou de structures discursives, mais ce sont également des actions langagières en coopération, définies par un but, une orientation, un enjeu pragmatique, qui instaurent des positions discursives, un type de rapport à l'interlocuteur et une façon de se poser soi-même comme locuteur, différente quand on explique ou quand on demande ou quand on argumente. Certaines renvoient à des genres culturels très spécifiques: la conférence, l'exposé ou le commentaire de texte par exemple; d'autres renvoient à des conduites plus «primitives », moins codées (codifiées) : questionner, nommer, définir, expliquer, comparer, confronter, raconter, qui elles-mêmes peuvent se codifier en genres culturels plus normés (les « discours explicatifs », les « discours argumentatifs », etc.).

Au cours de cette interaction, l'un des deux garçons (E : 20 ans) explique à l'autre (F: 22 ans) une équation mathématique. Les deux garçons sont d'origine sérère par le père, et mankagne par la mère. E parle le wolof, le français et comprend le créole, il a fait des séjours en Casamance dans la famille de la mère et il a suivi une partie de ses études au séminaire de Kaolack. F parle le wolof, le français, le créole et comprend le mankagne. Il est resté trois ans en Casamance dans la famille de sa mère, c'est là qu'il a appris le créole et « un peu » de mankagne. Ils vivent à Dakar depuis une dizaine d'années. Le wolof est la langue qu'ils ont déclaré avoir parlé en premier, puis le français; ils se considèrent bilingues wolof-français. Dans cette interaction, le code non marqué selon l'acception que lui réserve Myers Scotton est le français (voire une alternance français-wolof avec une nette prépondérance du français). Dans les entretiens réalisés avec les membres de ce groupe de jeunes ainsi que dans les notations de choix de langues réalisées à partir d'observations effectuées par un des interlocuteurs de cet échange, le français apparaissait comme la langue normalement attendue lors des devoirs et plus généralement au cours des interactions liées à la scolarité et à l'avenir professionnel.

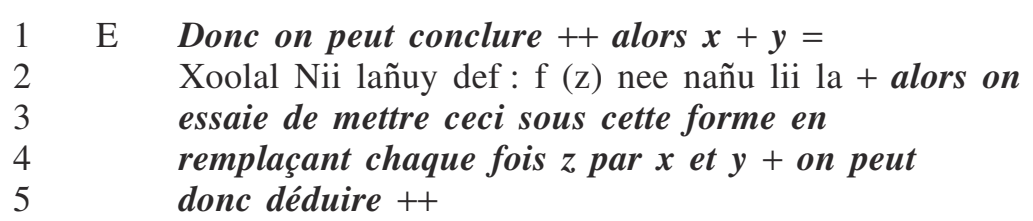




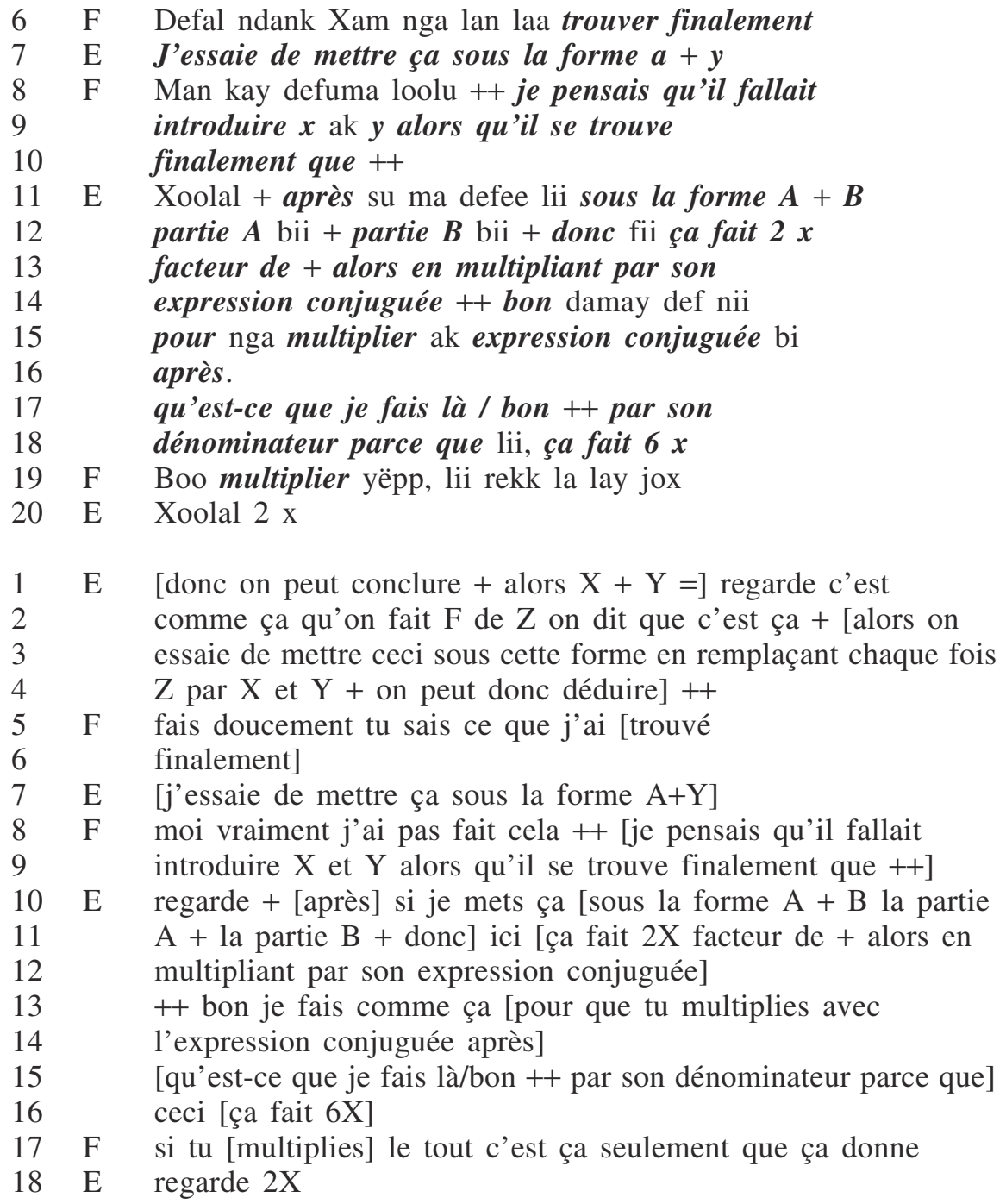

Les conduites discursives utilisées dans cet extrait ont une visée essentiellement explicative : un locuteur $\mathrm{E}$ fait comprendre à son interlocuteur $\mathrm{F}$ ce qu'est un certain objet (ici une équation mathématique) en le décrivant, en l'analysant devant lui ou en explicitant certains éléments ou aspects. La situation d'explication orale laisse place à une régulation permanente du discours explicatif en fonction des besoins de l'interlocuteur : il peut interrompre celui qui explique, lui poser des questions, demander des précisions, reformuler ce qu'il croit avoir compris.

$\mathrm{Au}$ début de l'interaction, au cours de la première intervention de $\mathrm{E}$, les segments d'énoncés en français reprennent les étapes constitutives de 
l'explication avec des marqueurs ou connecteurs logiques du type «donc, alors, ... » qui structurent le discours, jouent leur rôle d'éléments cohésifs, et organisent sa progression et la mise en relation des éléments. L'emploi réitéré de on souligne le caractère de discours explicatif à dominante objective. Le passage au wolof (1.2) marque une autre orientation discursive, et un déplacement énonciatif, à travers le glissement du « on » à «tu » : «xoolal » regarde, le locuteur interpelle et s'adresse directement à l'interlocuteur en redoublant d'une certaine façon l'explication, en la personnalisant et en la commentant. À nouveau, l'explication objective — on pourrait la qualifier de didactique ou de magistrale ou encore de «monologique », en tant que close sur elle-même - reprend en français à la ligne 2 à partir du marqueur de relation «alors » jusqu'à la ligne 5. L'intervention de l'allocuteur nous ramène à un autre plan énonciatif et interrompt cette explication magistrale (passage au «tu») et s'amorce à partir d'un énoncé en wolof «defal ndank... ». Cette intervention se place sur le plan d'une certaine subjectivation, elle sollicite une demande d'explicitation, et donc une relation plus personnelle. L'intervention qui suit (lignes 8-11) du même locuteur ( $F$ : celui à qui est adressée l'explication) est intéressante parce que le passage d'une langue à l'autre dédouble également les plans d'énonciation : le premier énoncé en wolof est un commentaire adressé à lui-même "man kay » alors que le second, en français, est un commentaire également mais il s'inscrit dans le discours explicatif et témoigne ici de la compréhension et de l'adhésion de $\mathrm{F}$ au discours d'autrui : "Je pensais que...alors qu'il se trouve finalement...»

L'intervention 11 de E est plus complexe : les segments d'énoncés en français et en wolof ne sont pas nettement délimités par des frontières syntaxiques précises et les deux langues y apparaissent plus étroitement mêlées : «après su ma defee » (1.11) «pour nga multiplier » (1. 15). Mais à nouveau on y relève des interpellations ou des commentaires adressés à soi ou à l'autre en wolof «xoolal...su ma defee...damay def nii... » (1. 11-14) ainsi que des éléments objectifs et des marqueurs de l'explication en français «après, donc, alors, parce que » (1. 11-18). Cette alternance marque aussi deux voix : une parole qui s'inscrit dans la logique d'un discours à tendance « monologique » et une autre qui commente ce discours et interpelle autrui (1. 19-20). La présence de la relation à soi ou à l'autre est massivement marquée par des segments d'énoncés en wolof.

Le passage du code non marqué (français) au code marqué (wolof) dans cette interaction traduit en quelque sorte, pour reprendre la terminologie et l'analyse de C. Myers Scotton (1993), un changement de droits et d'obligations entre les interlocuteurs et un autre niveau dans la relation interpersonnelle. Ces déplacements énonciatifs et discursifs que scande l'alternance du français et du wolof ne sont pas sans faire apparaître également, avec l'effet d'amplification propre au code mixte, «l'hétérogénéité » à la fois constitutive et montrée du sujet (Authier-Revuz 1995) et peut-être peut-on esquisser 
un parallèle, à un autre niveau d'analyse, avec deux des fonctions discursives de J. J. Gumperz (1989) : la fonction de qualification/modalisation du message et la fonction de personnalisation vs objectivation que nous avons évoquées plus haut (prise de position du locuteur sur l'importance relative des informations qu'il transmet dans son message et différence d'implication du locuteur par rapport à son message).

Cette forme de discours mixte est assez représentative de l'émergence d'un «parler bilingue » à Dakar, socialement reconnu et légitimé par des classes sociales qui sont, de par leur statut (de «lettrés»), considérées comme détentrices d'une certaine norme.

Le deuxième extrait est un fragment d'un enregistrement effectué lors d'une partie de jeux de billes entre deux garçons de 8 et 13 ans (A et C) et une fille de 11 ans (B). Nés à Dakar, de père sérère et de mère wolof, ils parlent le wolof et le français. La langue habituellement utilisée lors des jeux est le wolof.

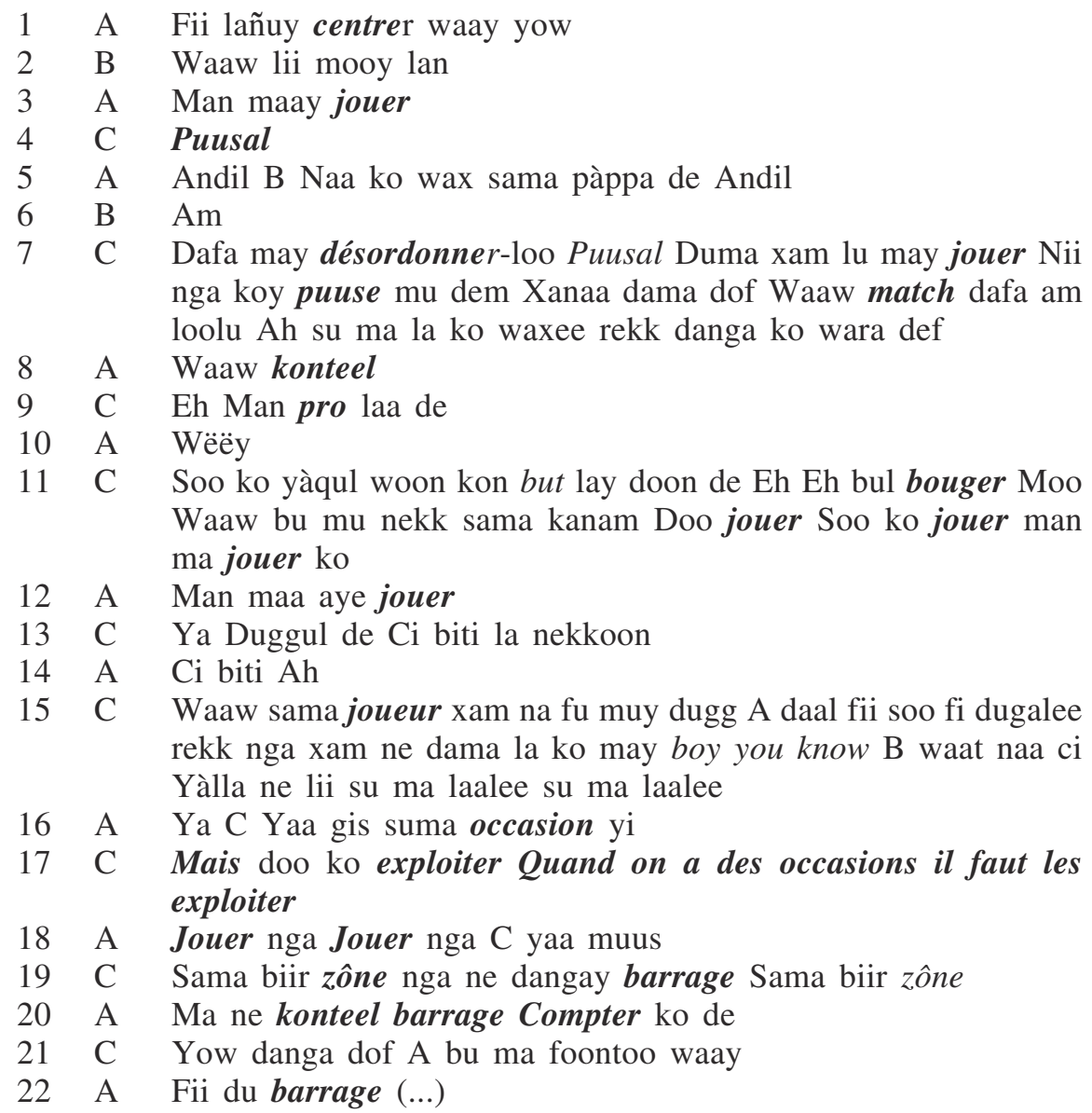




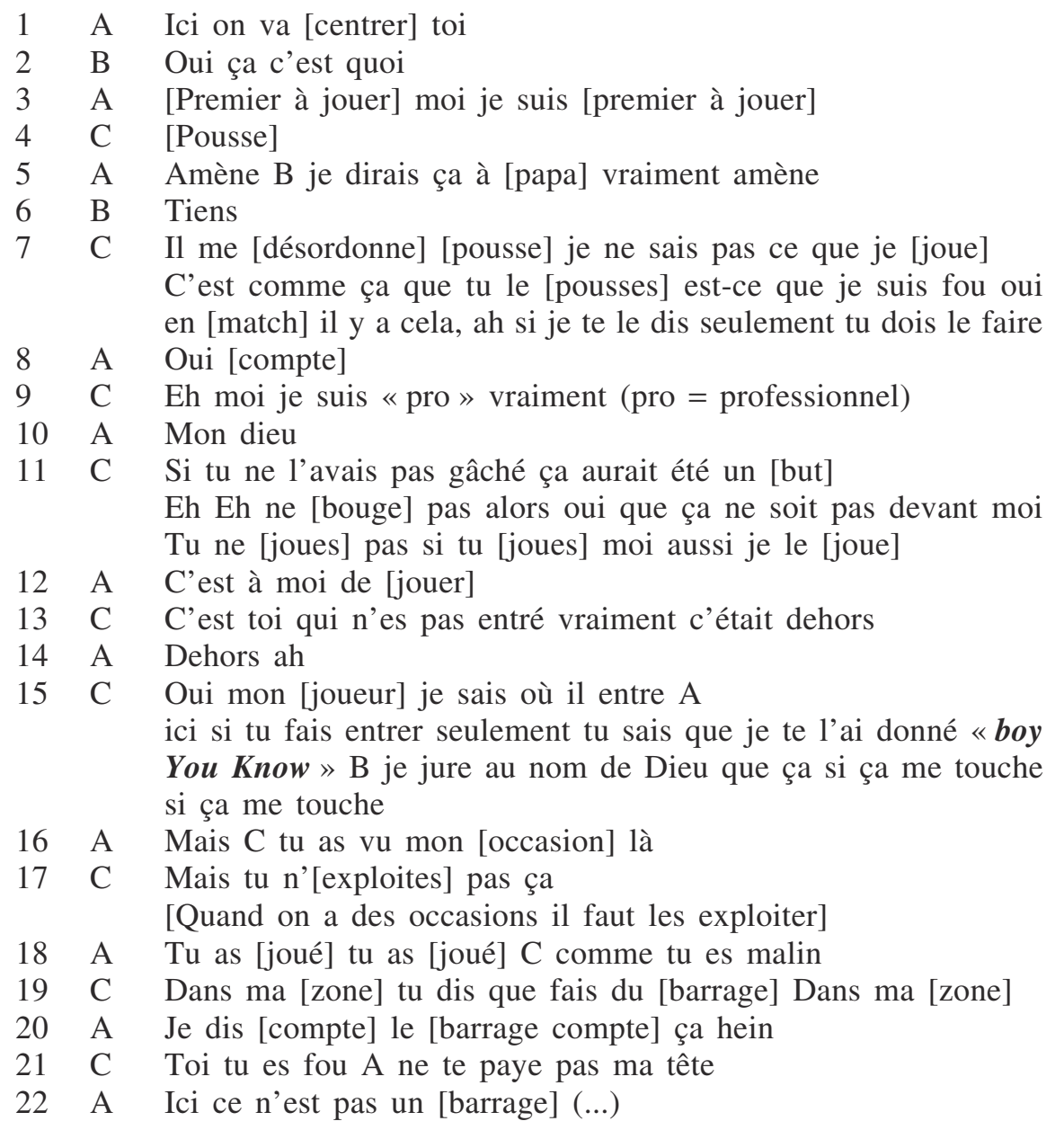

Cet extrait est à rapprocher de la partie de cartes enregistrée à Ziguinchor. La langue de base de l'échange est ici le wolof et le français apparaît sous la forme de lexèmes (verbaux ou nominaux) isolés et plus ou moins intégrés phonologiquement et morphologiquement au wolof : «konteel, puusal, centrer, joueur... ", à l'exception d'un segment d'énoncé plus long qui s'insère dans le discours sous forme de commentaire à valeur de citation : "Quand on a des occasions, il faut les exploiter» (1. 18), et nous renvoie aux fonctions discursives et sémantiques de l'alternance analysées dans le premier extrait. Cet énoncé est prononcé par le plus âgé des trois enfants. L'ensemble présente une grande unité linguistique tout au moins dans les procédés formels d'intégration des mots français au wolof. Le langage utilisé dans cet extrait a une fonction essentiellement référentielle (on pourrait le qualifier de «discret» par rapport aux composantes de la situation de 
communication); il s'agit essentiellement de nommer des objets ou des actions en cours, de redoubler par la parole les composantes du jeu et la part d'implicite dans cet échange est importante. L'interaction verbale accompagne et souligne les gestes des joueurs et les mouvements des billes. Le discours n'a pas une visée explicative et argumentative dominante comme dans le premier extrait; il y a, certes, des conduites discursives « primitives» (au sens défini plus haut) telles que ordonner, questionner, argumenter, réfuter... - on pourrait parler plus précisément ici d'actes de langage selon l'acception qu'en donne C. Kerbrat-Orecchioni (1990 : 229) en analyse conversationnelle - mais ces actes ou conduites ne sont pas marqués dans le discours par un passage du wolof au français. Cette variété utilisée ici par les jeunes est assez représentative de ce que certains nomment le wolof « urbain », langue à base wolof dans laquelle s'insère des éléments d'autres langues, ici l'anglais apparaît également (but, you know).

Ces deux «variétés » de discours mixte, présentes à Dakar, représentent des actualisations possibles, dans différents contextes communicationnels et interactionnels, de la réalité plurilingue, en même temps que la manifestation d'une identité personnelle autant que collective. Il faut également souligner qu'émerge, à Dakar, une variété linguistique mixte, qui à la fois se diffuse dans plusieurs espaces sociaux et témoigne, pour certains groupes de locuteurs, d'une identité «dakaroise».

\section{Le discours mixte à Ziguinchor}

Si, à Dakar, l'alternance wolof/français peut symboliser l'identité dakaroise, à Ziguinchor, la langue de groupe reste très présente. Elle est la langue de base des mélanges observés chez de jeunes locuteurs scolarisés ; le français, le wolof et éventuellement d'autres langues ou dialectes locaux sont les langues imbriquées ( embedded languages », au sens de C. Myers Scotton).

Nous disposons de deux corpus de longueurs inégales, recueillis lors de situations informelles, récurrentes de la vie quotidienne de jeunes Diola d'un quartier périphérique de Ziguinchor, où les Diola sont majoritaires. Des extraits de ces interactions ont été déjà présentés (Juillard 1995: Annexe 6 \& chap. 2 : 112-117). Il s'agit d'une partie de cartes (170 tours de parole) et d'une discussion autour du thé (167 tours de parole). Les enregistrements ont été transcrits et traduits plus tard, sans qu'on ait pu identifier exactement qui parle à chaque tour de parole. Chaque tour de parole correspond à un locuteur différent du précédent sans qu'on ait pu, a posteriori, préciser son identité.

Nous proposons une analyse des mélanges relevés sous deux aspects. Premièrement, l'alternance est révélatrice d'une négociation sur l'identité partagée. Deuxièmement, les formes spécifiques de l'alternance relevée chez ces jeunes gens de Ziguinchor révèlent l'importance maintenue d'une langue 
de base de l'échange, laquelle se trouve être le plus souvent la langue emblématique du groupe d'appartenance. On constate cependant une certaine souplesse du contact des langues et un changement de la langue de base des échanges, en relation notamment avec les changements thématiques.

\section{La langue (et le mélange) comme outil du débat sur l'identité}

La prise en compte et la mise en relief de l'alternance linguistique par les co-actants leur permet de se situer respectivement au sein d'un espace social, parfois conflictuel, parfois consensuel, qui les lie.

Il se trouve que l'enregistrement de la partie de thé démarre sur une discussion sur l'usage des langues dans le groupe de jeunes pairs ${ }^{10}$.

La partie de thé : c'est le début de l'enregistrement et de la discussion entre eux.

$1 \quad \boldsymbol{w a w}^{11}$

oui

2 unolen u melã ze kasãykenak kujolak c'est la même chose !

tu peux mélanger les manières de parler, en diola c'est la même chose

3 le grawul, usãy kenak kujolaku

ce n'est pas grave, nous pouvons parler diola

4 bu kane? bu kane?

comment ça se fait ? comment ça se fait?

5 ujamut kujoloeyoeku ?

tu ne comprends pas diola?

6 oña kasãyken man buku...

il parle pour que eux....

10. Dans les extraits présentés, les enregistrements ont été réalisés à micro caché. À Dakar, ils ont été réalisés par un des membres du groupe des jeunes et des adolescents, ceux-ci avaient toutefois été prévenus de la possibilité d'un enregistrement lors de leur conversation. À Ziguinchor, ils ont été réalisés par un jeune Diola ami des jeunes qui ont l'habitude de se réunir quotidiennement, en fin de journée, pour jouer aux cartes ou boire du thé. La première rencontre a réuni des jeunes Diola, habitants de longue date du même quartier. La deuxième rencontre a réuni 7 jeunes Diola; la majorité d'entre eux habitent le quartier depuis longtemps; trois d'entre eux sont nés à Dakar et sont venus habiter le quartier à date récente; de ce fait, ils parlent moins bien le diola que les autres, avec lesquels ils peuvent tenter de s'exprimer en wolof. Leur présence peut expliquer cette négociation sur la langue à utiliser tous ensemble. Les conversations enregistrées sont spontanées et n'ont fait l'objet d'aucune incitation, thématique ou formelle.

11. Le diola est transcrit en caractères simples; le wolof en caractères italiques : les alternances vers le français sont soulignées; les emprunts au français sont en caractères gras ; les emprunts à l'anglais sont en caractères gras soulignés. 
7 mais kooku grawul

mais cela n'est pas grave

8 usãyken kujola

tous ensemble, parlons diola

9 yepp bennoeloe!

tout ça, c'est pareil !

10 hã, hã, kujolaku di benarey !

non, non, moi, je veux opter pour le diola !

11 konom mustafa je me prénomme Moustafa

12 sãyken ku wolofay, sãyken kujolaeyk tu moe konoemu que tu t'exprimes en wolof, ou en diola, c'est exactement la même chose

13 usãyken kama! useni, komitemi, kolatier ! alors, parle! Houssein, je vais te critiquer, je vais te critiquer!

14 Ha, kubalãn, bala ja a balãybalãy (rires) jat (rires) ah ! il résiste, Bala, aujourd'hui, est résistant aujourd'hui

15 hokammarẽ̃ lui, il fait cela pour qu'il ramollisse (= pour l'humilier)

$16 \quad l \varepsilon$ mi kugabajije moomu n'est-ce-pas que c'est vous deux qui avez décidé comme ça (la suite des choses)

17 he boy, inje ! hé, mon pote, c'est moi !

La construction de l'identité collective s'inscrit ici au sein de rapports interpersonnels d'imitation, de réitération, de catégorisation, de différenciation, qui se succèdent d'un tour de parole à l'autre. L'alternance linguistique est l'un des moyens d'exprimer l'univers de référence commun. Dès les premiers tours de parole émerge une réflexion collective sur les deux langues qui vont ensuite alterner dans la discussion, le diola et le wolof. Elle prend la forme de commentaires en diola et en wolof, parfois modélisés dans le sens d'une recherche de consensus, puis d'une joute pour décider de la prise de parole en langue, en l'occurrence en diola.

Le diola est d'emblée présenté comme une langue mélangée : «/ujolen u melãze kasãykenak kujolak/ c'est la même chose » = « tu peux mélanger les manières de parler (i.e. les dialectes), en diola c'est la même chose ! » dit le premier intervenant. Pour exprimer cette notion de mélange, il recourt à un emprunt français intégré et à une alternance codique avec le français. L'idée que toute forme est équivalente reviendra, plus loin, à propos du wolof et du diola (tp 12) : «/sãyken ku wolofay, sãyken kujolaeyk tu mœ konœmu /» = «que tu t'exprimes en wolof, ou en diola, c'est la même chose ». La modélisation qui vise à établir un consensus entre des parties adverses en limitant la portée de l'alternance s'exprime d'abord en français (tp 2), puis en wolof (tp 7 et 9), enfin en diola (tp 12). Si l'identité collective 
qui rassemble ces jeunes n'est pas explicitement désignée, l'usage, à deux reprises, de la forme verbale au pluriel /usãyken ku jola/ qu'on peut au mieux traduire par «tous ensemble, parlons diola», renvoie à un collectif de la parole, négocié par la parole, qui fonctionne implicitement comme référent de l'adéquation langue/identité «je suis diola, je parle diola ${ }^{12}$. L'identité se révèle donc grâce au positionnement que permet la prise de parole en langue $\mathrm{X}$ ou $\mathrm{Y}$.

Plus loin, dans la même interaction, les identités sont désignées explicitement, lors d'un commentaire sur la forme utilisée : /kati foñi kone aw eduk/ = «Ceux de Fogny ont dit: toi tu vois ». Celui qui vient de parler en utilisant la forme /ejuk/ a été repris par le suivant qui l'a corrigé : /eduk/ et l'a, ce faisant, situé dans un collectif dialectal/régional. Comme le diolaFogny est plus parlé à Ziguinchor que les autres dialectes, d'après les témoignages, son usage est usuellement non marqué. Cet usage aurait donc pu ne pas être relevé, mais dans le procès discursif, connoter explicitement l'origine rurale a permis une moquerie à l'égard de ceux qui vont voir les filles.

La discussion tourne autour du fait d'aller se coucher ou d'aller voir les filles.

49 mante ejuk bajanabu /kone ay emmi kamoraku a loberit / inje ejow kamor bey jow beyduk bajanabu

peut-être aller voir les filles / On a dit celui qui va se coucher ne prévient pas / Moi je vais me coucher, je vais aller voir les filles.

50 eynow ejuk bajanabu

le gars va voir les filles

51 kati foñi kone aw eduk ceux de Fogny ont dit: toi, tu vois

52 iyo man ter ah bon peut-être

53 iyo usaafi bo / pambu yañora, ula ja rekk, tendimœne sẽygor oui, salue-les moi. On peut se rencontrer là-bas, on n'a qu'à y aller sûrement, les habitants de Tendimane, faire l'amour.

Dans cet extrait, dont la langue commune est le diola, l'accent est mis sur le marquage d'éléments dialectalisés pour scinder le collectif et cibler un segment périphérique du collectif. Retenir la marque a permis de renégocier

12. La langue diola présente une grande diversité dialectale. Le Fogny est la région située au Nord du fleuve Casamance et les dialectes qu'on y parle présentent des différences avec ceux du Sud. Le dialecte du Fogny est prépondérant en ville, semble-t-il. 
l'identité diola en distinguant ceux d'ici, les citadins, de ceux de là-bas (Fogny et Tendimane), les ruraux.

Dans la partie de cartes, la majorité des tours de parole sont en diola, parfois ponctués d'emprunts au français et d'alternances avec le français et plus rarement le wolof. À un moment surgit une appréciation sur un joueur chanceux, appréciation qui s'exprime en manding, pendant cinq tours de parole (cf. exemple ci-dessus).

Le manding est une langue que parlent les Diola du quartier, grâce à la cohabitation avec les Manding. Elle n'est manifestement pas d'un usage si courant entre eux, cependant. Mais son usage est connoté du prestige associé à l'identité manding, en ville et dans la région. La langue manding est, d'ailleurs, la plus véhiculaire des langues de la région. Les commentaires, en diola, qui suivent ces prises de parole en manding, ainsi que les rires qui les accompagnent, contribuent à la construction de l'identité collective : «Ah! Nous sommes en train de nous exprimer en manding ici / Ah! C'est positif. »

Le fait d'exprimer en diola le caractère positif de l'usage du manding entre Diola leur permet de signaler leur solidarité intra-groupe : la représentation consensuelle du chevauchement socioculturel est exprimée ici par le contact des langues faisant partie de leur répertoire collectif.

L'alternance linguistique est donc un outil de marquage identitaire, qui peut parfois être relevé de commentaires métalinguistiques à des fins consensuelles ou discriminantes. La plupart du temps, cependant, les alternances fonctionnent sans être relevées. Elles prennent des formes récurrentes qui sont aussi, sur la durée, des marqueurs identitaires.

\section{Formes et thématiques associées}

Dans l'un et l'autre corpus, l'alternance linguistique la plus fréquente consiste dans le changement de langue qu'opère un locuteur donné, lors de sa prise de parole. Il s'agit donc principalement d'une alternance extraphrastique. Cependant la forme des deux interactions relevées auprès de jeunes Diola de Ziguinchor est assez différente, puisque le diola est prépondérant dans la première et l'est moins dans la deuxième, où l'alternance avec le wolof est beaucoup plus saillante.

La partie de cartes présente plus d'emprunts et d'alternances vers le français, que vers les autres langues du répertoire linguistique utilisé lors de cet échange (diola, wolof, français, manding). La langue de base de l'interaction est probablement une forme véhiculaire ou urbaine du diola, présentant, comme d'autres langues utilisées en ville, un grand nombre d'emprunts au français insérés morpho-syntaxiquement et intégrés phoniquement. Ces emprunts ne sont pas balisés. Ce sont majoritairement des verbes utilisés avec un sémantisme un peu particulier, lié à la situation du jeu ou bien propre au discours des jeunes urbains : /tire/ $=$ «miser »; 
/poñore/ = «s'empoigner »; /berze/ = « héberger » (au sens de prendre la carte), /mõnte/ = «avancer ». Pour le reste, on relève des emprunts établis relatifs au jeu de cartes (valet, as, roi, pique, cœur, carreau, trèfle, chance, fort, faible, des chiffres). Certains emprunts utilisés par ces jeunes pourraient bien avoir transité par le wolof : en particulier /deplane/ « déconner ». Outre ces emprunts, les alternances en français (extraphrastiques surtout) témoignent d'une relative aisance bilingue, dans cette interaction dont le rythme de parole est très rapide.

Tours de parole : 17 à 22

17 No køre, c'est beaucoup de choses

Non, c'est le cœur, c'est beaucoup de choses

18 U teendiji pikey uyey / injebey seni ji pikey

Sers avec ce pique-là. Moi, je vais te donner un petit pique

19 C'est combien ? :

20 Non, parmi les deux piques

21 Soixante dix neuf, a

Soixante dix neuf, sûrement

22 U mõnte, jat paniri...

Si tu viens (si tu t'avances avec ta carte), aujourd'hui, je vais te manger...

Des énoncés en wolof urbain, plus rares (tpp. 44 et 46), s'insèrent dans un contexte diola, comprenant des bruitages spécifiques, des interpellations en diola, des phatèmes et autres ponctuateurs de discours, l'usage de proverbes diola, des rires constants :

Tours de parole : 42 à 46

42 ñe u gorormu u juka / manemmi miyu jinare

Bouge, affirme-toi pour qu' on voit ce que tu peux faire (avec

43 Wasa!

ta carte) / puisque c'est vous qui allez vers la victoire.

(= un bruit diola)

44 Ẽhẽ, bimoom, boy, buljapul dara

Sûrement, celui-là, boy, il n'a rien attrapé (= il a pris une mauvaise carte)

45 Ẽhẽ, ẽhẽ, ẽhẽ (bruit diola exprimant l'approbation et/ou l'attention, répété $5 / 6$ fois par plusieurs)

46 Xȭxaxee! boy! Mais, afer-bi baxul quoi !

(bruit diola exprimant un avertissement), boy, je veux bouger, mais l'affaire n'est pas bonne.

47 Non, non, non, prochaine fois !

La partie de cartes présente une grande unité de genre. La discussion est très animée; les paroles sont entremêlées, les répliques fusent. Ces 
jeunes jouent ensemble aux cartes tous les jours; il s'ensuit une certaine ritualisation perceptible dans la forme autant que dans le contenu. On n'observe pas de rupture interne; ainsi, lorsqu'un des participants raconte une anecdote, pendant qu'ils jouent tous et que la parole lui est souvent prise pour le jeu, il n'y a pas de changement formel conséquent: la langue de base des échanges reste du diola urbain, avec insertions d'emprunts au français. De plus, lorsqu'un locuteur change de langue lors de sa prise de parole (alternance extraphrastique vers français, wolof, voire manding), ce changement reste concomitant de l'univers de discours qui ne change pas; s'il est repris ensuite par d'autres locuteurs, l'alternance ne se maintient pas pour plus de quelques tours de parole, et la langue de base est rapidement réutilisée. Il en est ainsi pour quelques avertissements en manding, ou en wolof, lors d'un moment critique du jeu, et d'une très courte altercation en wolof. Celle-ci n'a duré que deux tours de parole :

90 dajga dof!

$\mathrm{Tu}$ es fou

91 yow la dof!

Tu es plus fou que moi!

Ou, encore, un rapide duo en français :

\section{Partie jouée!}

113 Ah oui, quand c'est lent

Hormis ces quelques apartés que soulignent les alternances, la discussion collective autour de la partie de cartes présente une remarquable unité discursive, thématique et formelle.

La discussion enregistrée pendant la partie de thé est beaucoup plus discontinue. La part du wolof augmente et concurrence progressivement et quantitativement la part du diola dans la conversation, au point que la langue de base de la discussion est alternativement l'une, puis l'autre.

La partie de thé rassemble des jeunes gens qui seront rejoints, à la mitemps, par une, puis deux jeunes filles. Dans la première partie, la discussion, démarrée en diola, à l'assentiment général, après une âpre négociation sur le choix de la langue à utiliser ensemble, se termine en wolof. Lorsque la discussion dérive sur les filles, la drague et le sexe, la part du wolof augmente et celle des tours de parole en diola diminue. Les tours de parole en français sont beaucoup moins fréquents que ceux en wolof, surtout avant l'arrivée des filles, qui se serviront de cette langue comme d'une voie de traverse, pour initier un changement thématique à leur avantage.

Dès le début de l'échange, différentes séquences thématiques successives ou imbriquées s'articulent et cette dynamique du sens est soulignée par les changements de langues :

- une discussion sur l'usage linguistique, en diola majoritairement, avec deux tours de parole en wolof et une alternance interne, du wolof 
vers le diola, alternances qui expriment une modélisation et la recherche d'un consensus ;

- puis, quelques échanges en wolof, relatifs à l'attente collective de l'un d'eux, Houssein, démarrent sur l'évocation de leur activité antérieure : regarder ensemble le feuilleton Santa Barbara à la télévision ;

- en diola : la confection du thé s'insère dans la discussion;

— on cherche Ndiaye : où est-il ? Il est allé draguer?

Ici s'insère l'extrait suivant :

Tours de parole : 27 à 42

27 Ndiaye, oh fãn afulot?

Ndiaye n'est pas sorti

(ici, quelques tours de parole en diola à propos de Ndiaye)

31 Ndiaye inje eregol man ajeu... basãy utuma

32 totey?

Ndiaye, je lui ai dit pour aller ici, là, pour draguer

À quel endroit?

33 ñow ban? now fan? ban ji ejow basan bey?

Aller où ? Chez qui ? Vous allez draguer à quel endroit?

Cette alternance vers le wolof, survenue après plusieurs tours de parole en diola, aura pour effet de relancer dans les tours de parole suivants une dynamique d'alternance entre les deux langues, avec cependant une dominante en wolof :

34 Mais boy, sa ataya bi dafa ki trop fort !

Mais, mon pote, ton thé là, il est trop fort !

35 dafa for-de!

Il est vraiment fort!

36 waxoon naala, Đgawañiwoon ggawañi ndox bi, mais ni dafa weex grawul, man-de, dax duma nelow tey, nelow tey.

Je te l'avais dit de diminuer, diminue l'eau, nous ne sommes que trois comme ça, mais ça c'est amer. Ce n'est pas grave, en ce qui me concerne, je ne vais pas dormir cette nuit.

37 jano pi mori, parce que te uye yati comme çà yo vraiment c'est... Parce que ce thé là comme ça vraiment c'est...

38 boy, du gañi drog la def

Boy, les camarades ont mis de la drogue dedans !

39 He ! He ! Oh, ah là là, grawul!

$\mathrm{He}$, he, oh, ah, là,là, ce n'est pas grave !

Une rupture thématique intervient, introduite par l'interpellation d'un participant, en diola : 
Eñab, bu du lobe?

La force tranquille, qu'est-ce que tu dis de beau?

41

Kasumay

Ça va

La discussion continue en diola : quelques échanges entre eux : «ça va ?», «ça va ? », «Ahmed, tu vas dormir ? »; « dormir » entraîne « se coucher », qui entraîne «aller voir les filles » et «faire l'amour»; cette progression thématique, accompagnée de sous-entendus, se déroule en diola, puis l'évocation plus précise, par l'un des participants, d'un souvenir à référent sexuel partagé, fait basculer la discussion en wolof majoritairement, du $54^{\mathrm{e}}$ au $72^{\mathrm{e}}$ tour de parole.

Globalement, la majeure partie des alternances entre les deux langues dominantes de cette première partie de la partie de thé, le diola et le wolof, fonctionne de manière consécutive : à des séquences d'un certain nombre de tours de parole en diola, langue de base, succèdent des séquences d'un certain nombre de tours de parole en wolof, langue de base. Le français se manifeste plus sous forme d'emprunts que sous forme d'alternances. Les énoncés en wolof comportent plus d'emprunts au français, dans ce corpus, que les énoncés en diola, qui n'en comportent que quelques uns. Dans les séquences en wolof, on relève des ponctuateurs de discours : « Mais, pourtant, je te jure, sans faute. » Et de nombreux emprunts spontanés, non balisés, émaillent les séquences en wolof (femme, /bor-yi/ «les bords = quartiers »/koñ-bi/ «le coin = la rue », affaire, /ga-ñi/ «les gars = camarades », drogue, fort, faute, temps, samedi, téléphone, tourner: tous emprunts intégrés morpho-syntaxiquement). De même que la locution /grawul/ «ce n'est pas grave », tous ces emprunts font partie intégrante du vocabulaire des jeunes bilingues, lorsqu'ils parlent wolof.

La seconde partie débute par l'arrivée d'une jeune fille du quartier F, à la recherche de son ami Ali, qui modifie le tour de la conversation : les échanges reprennent en diola; mais dès que l'un des jeunes gens évoque les succès féminins d'Ali, le wolof est réutilisé par tous et $\mathrm{F}$ embraye, contente de se moquer avec les autres, Indax dafuma daral «car il ne m'a rien fait »! Puis ils jouent tous ensemble, en français, à la devinette sur le prénom de l'amie d'Ali ; c'est F qui a lancé cette langue, en ironisant sur les conquêtes d'Ali, et tous l'ont suivie et sur le choix de la langue et sur la thématique. Différents échanges à 2 ou 3 interfèrent, avec changements de thématiques et de langues; une seconde jeune fille arrive: salutations. Puis le jeu de devinette repris, en français, par $\mathrm{F}$, les mène à une certaine Penda. Il est alors question, entre hommes et en wolof, des charmes de Penda et de la draguer. F intervient une fois en wolof, de manière consensuelle, mais quelques tours de parole plus tard, elle change de langue pour leur signifier qu'ils sont déjà en retard sur leurs projets de drague : 
- Babaj aje amanda katoporo (Il y a déjà quelqu'un qui est en train de s'en occuper (= de Penda)).

- Réaction bruitée collective : xõnxaxee!

Toute la discussion reprend en wolof, mais la jeune fille, en diola, coupe court et quitte le groupe ; tous ensemble essayent de la retenir, en diola :

— Nõ, jol, jol, jol (Non, viens, viens, viens ! (= pour tout rééplucher au sujet de Penda).

Les jeunes gens reprennent toute la discussion, en wolof surtout.

L'imbrication des séquences thématiques et des langues est, dans toute cette partie, beaucoup plus dynamique et plus souple. Les locuteurs se coupent souvent la parole, des échanges ne concernent parfois que deux ou trois personnes et les changements de langues utilisées par les uns ou les autres (diola, français, wolof) sont fréquents. Le glissement vers des échanges collectifs majoritairement en wolof et sur une durée plus longue, est lié à la recherche d'un consensus, ici surtout masculin, autour d'évocations à connotation sexuelle.

La comparaison des deux interactions, partie de cartes et partie de thé, fait donc ressortir des habitudes linguistiques un peu différentes, selon le genre discursif : rituel d'une partie de cartes ou discussion à bâtons rompus et avec incidentes sur thématiques, elles aussi, ritualisées jusqu'à un certain point. Le diola est prépondérant dans la première et l'est moins dans la deuxième, où l'alternance avec le wolof est plus saillante. Des éléments de français sont toujours présents sous la forme préférentielle d'emprunts spontanés, mais également sous la forme d'alternances codiques ; cette présence se situe sur des plans de marquage différents; il peut s'agir d'une modernisation de la langue emprunteuse par adoption et intégration de signes provenant de la langue qui connote le plus cette modernité ; il peut également s'agir de véritables choix de langue, par mouvements de courte durée et implication personnelle ou collective, de la part de ces jeunes scolarisés qui partagent cette expérience et la langue qui y est associée. La durée des séquences en français n'est jamais aussi longue que celle des séquences en diola ou en wolof. Il y a donc un effet de marquage d'un autre type que pour le diola ou le wolof.

L'effet du français « à l'intérieur » de la langue emblématique du groupe (diola urbain de la partie de cartes) ou des langues africaines en compétition (diola et wolof urbains de la partie de thé) permet aux locuteurs de se situer dans un univers à choix multiple. La présence d'éléments de français imbriqués dans les énoncés en wolof, plus dominante dans la deuxième interaction, renforce, à notre avis, la compétition entre les deux langues africaines utilisées concurremment, et accuse la connotation de modernité pour l'usage du wolof, en relation avec des séquences thématiques pour lesquelles l'usage du diola est, d'après les entretiens effectués, ressenti par les usagers comme malséant. L'imbrication des langues du répertoire rend manifeste la prédominance du diola et du wolof comme langues alternatives 
exprimant le collectif ; mais si, par moments, du wolof peut s'insérer dans des échanges en diola, l'inverse est moins fréquent. Le français reste d'un usage mesuré, mais constant, et généralement s'imbrique au sein d'échanges en l'une et l'autre langue, et surtout celle qui semble être la plus porteuse du collectif, au moment précis où elle est utilisée. Elle peut également servir de troisième voie, lorsqu'on veut contourner le consensus.

L'évolution en cours à Dakar se situe dans le sens de l'émergence d'une variété linguistique distincte dont témoigne le premier extrait présenté. La variation du discours mixte s'organise alors autour d'actualisations discursives diverses ou de registres de langue disponibles et mobilisés par le locuteur dans telle ou telle situation de communication. Cette variété s'organise essentiellement autour de l'alternance du wolof et du français, ce qui ne me semble pas être le cas à Ziguinchor, pour le moment, dans la mesure où dès que le consensus entre les jeunes se disloque, on voit réapparaître les langues de groupe. Quoique moins fluide et moins imbriqué que dans les corpus relevés à Dakar, le mélange des langues pratiqué par les jeunes Diola de Ziguinchor témoigne d'une grande flexibilité linguistique, qui est l'indicateur actuel d'une variabilité linguistique présente à Ziguinchor de longue date. Cette flexibilité linguistique est aussi présente à Dakar, mais elle s'organise dans un espace moins nettement plurilingue, la région ayant été depuis très longtemps majoritairement peuplée de locuteurs wolophones.

Les jeunes Diola de Ziguinchor s'identifient à la langue emblématique du groupe d'appartenance et à celle dont l'usage commun les rassemble tous, le wolof. D'autres références, secondaires, peuvent surgir également, qu'il s'agisse d'autres langues régionales partagées ou de particularismes dialectaux. Le français, toujours présent, est plus en retrait, mais leur permet également de manifester une appartenance commune partagée, en référence au monde scolaire, comme le wolof. Les mêmes constatations peuvent être faites à Dakar, pour les jeunes, quelle que soit l'origine des locuteurs, en ce qui concerne le wolof et le français ; la langue de groupe joue «probablement » (nous n'avons pas de corpus en nombre suffisant pour l'attester) un rôle différent selon les groupes d'appartenance ; certains, tels les Toucouleurs, apparaissant plus conservateurs et organisés autour de réseaux sociaux denses (Milroy \& Milroy 1985). Les jeunes Diola à Ziguinchor appartiennent à des réseaux sociaux, agissant comme une force conservatrice qui vient ralentir la diffusion des changements linguistiques. Certes, l'usage du wolof et du français représente une ouverture, un changement par rapport à des usages plus catégoriques de la langue de groupe, que ces jeunes sont susceptibles d'actualiser lorsqu'ils se retrouvent face à leurs aînés ou face à des ruraux. La flexibilité des usages mélangés, entre eux, implique qu'une certaine «modernisation » du parler de base est à l'œuvre, sans pour autant 
qu'il soit abandonné. Il n'est pas exclu qu'à l'âge adulte, la polarisation ethnique reprenne le dessus. Il n'est pas non plus exclu que d'autres langues sénégalaises (ou étrangères) soient apprises ultérieurement. Il a été attesté, par de nombreux témoignages, que l'usage du wolof, à Ziguinchor comme à Dakar, augmente en proportion du brassage ethnique et du regroupement des jeunes entre eux. Mais à Ziguinchor, il est toujours possible de revenir à l'usage des langues de groupe, lorsque le consensus éclate et que les identités ethniques (voire ethnico-villageoises) resurgissent, alors qu'à Dakar la pratique des langues de groupe dans des réunions de jeunes semble moins présente, les quartiers ne présentant pas des regroupements ethniques importants ou tout au moins suffisants pour lutter contre les phénomènes de dilution ethnique.

\section{BIBLIOGRAPHIE}

Authier RevuZ, J.

1995 Ces mots qui ne vont pas de soi. Boucles réflexives et non cö̈ncidence du dire, 2 t., Paris, Larousse.

BAKHTINE, M.

1984 Esthétique de la création verbale, Paris, Gallimard.

Bensalah, A.

1998 «L'alternance de langues comme marqueur du changement des genres discursifs et de l'accentuation de l'intersubjectivité », in A. QuEFFÉLEC, ed., Alternances codiques et français parlé en Afrique, Aix-en-Provence, Publications de l'Université de Provence.

BOUTET, J.

1994 Construire le sens, Berne, Peter Lang.

DAFF, M.

1995 Le français mésolectal oral et écrit au Sénégal : approche sociolinguistique, linguistique et didactique, Thèse de Doctorat, Dakar, Université Cheikh Anta Diop.

Diouf, J.-L.

1991 «Quelles langues parlent-ils à Dakar?», Communication au colloque international «Des langues et des villes », Paris, Didier Érudition : 227-236.

Doneux, J.-L.

1978 «Les liens historiques entre les langues du Sénégal », Réalités africaines et langue française, 7. 
DREyfus, $\mathrm{M}$.

1995 Le plurilinguisme à Dakar. Contribution à une sociolinguistique urbaine, Thèse nouveau régime, Paris, Université René Descartes-Paris V.

1999 «Parlers urbains », LIDIL, $19: 29-41$.

DumONT, P.

1973 Les emprunts du wolof au français, Dakar, Centre de linguistique appliquée de Dakar.

GUMPERZ, J. J.

1989 Sociolinguistique interactionnelle. Une approche interprétative, Paris, L'Harmattan.

JuillaRd, C.

1995 Sociolinguistique urbaine. La vie des langues à Ziguinchor (Sénégal), Paris, Éditions du CNRS.

Juillard, C. \& Wald, P.

1994 «Présentation du numéro thématique sur le plurilinguisme au Sénégal », Langage et Société, 68 : 6-10.

Kerbrat-Orecchioni, C.

1990 Les interactions verbales, Paris, A. Colin.

MANESSY, G.

1995 Créoles, pidgins, variétés véhiculaires. Procès et Génèse, Paris, Éditions du CNRS.

Milroy, J. \& Milroy, L.

1985 «Linguistic Change, Social Network and Speaker Innovation », Journal of Linguistics, $21: 375$.

Myers SCOTTON, C.

1993 Social Motivations for Code Switching. Evidence from Africa, Oxford, Clarendon Press.

NDAO, P. A.

1996 Contact de langues au Sénégal. Étude du code switching wolof français en milieu urbain: approches sociolinguistiques, linguistiques et pragmatiques, Thèse de doctorat d'État, Dakar, Université Cheikh Anta Diop.

NicOLAÏ, R.

1987 «Question sur le code switching conversationnel », communication au colloque contacts de langues : quels modèles?, Nice, dactylographié.

PARKIN, D.

1977 «Emergent and Stabilized Multilingualism: Polyethnic Peer Groups in Urban Kenya », in H. GILES, ed., Language, Ethnicity and Intergroups Relations, London-New York, Academic Press : 185-210. 
REINSCH, M.

1994 Colonial Dialogue: Language Mixing in Dakar, Senegal, Doctorat, Washington, University of Washington.

SwIGART, L.

1990 «Wolof: langue ou ethnie ? Le développement d'une identité nationale», Actes du colloque, Des langues et des villes, Paris, ACCT.

1992 Practice and Perception: Language Use and Attidues in Dakar, Thesis, Washington, Washington University.

THIAM, N.

1994 «La variation sociolinguistique du code mixte wolof français à Dakar : une première approche », Langage et Société, 68 : 11-32.

1998 «La coexistence du français et des langues nationales en contexte urbain sénégalaisé », Deuxièmes journées scientifiques du Réseau sociolinguistique et dynamique des langues de l'AUPELF-UREF, Rabat, 25-28 septembre 1998, non publié.

WALD, P.

1994 «L'appropriation du français en Afrique noire une dynamique discursive », Langue française, 104 : 115-124.

\section{RÉSUMÉ}

L'article développe le point de vue selon lequel I'alternance linguistique correspond à une option du répertoire plurilingue des jeunes urbains scolarisés, au Sénégal. L'analyse d'interactions relevées tant à Dakar qu'à Ziguinchor, met en évidence des modalités différentes du discours mixte, selon les lieux (la capitale, au centre, la ville régionale, à la périphérie) et selon les genres discursifs (discussions de groupes, discours explicatif à deux, parties de jeux à plusieurs). On a constaté que l'alternance linguistique renforce la valeur perlocutoire des échanges argumentatifs insérés dans les discussions. On assiste à Dakar à l'émergence d'une variété mixte wolof-français ; à Ziguinchor, le mélange de langues témoigne à la fois de l'identification plurielle des usagers et du maintien de l'identité ethnique : le discours mixte se caractérise par la présence d'alternances (extra-phrastiques principalement) et d'emprunts au sein d'une langue de base, qui peut elle-même alterner : langue du groupe d'appartenance ou wolof.

\section{ABSTRACT}

Language-switching in the Everyday Lives of School Children in Dakar and Ziguinchor (Senegal): Variation on the Use of French and Wolof. - The analysis of language interactions between children in Dakar and Ziguinchor (Senegal) sheds light on the different sorts of mixed uses of language, depending on the place (the capital in the center, a regional city on the periphery) and the discursive genres (group discussions, conversations between two persons, or games with several players). Language-switching reinforces the perlucotor value of the argumentations exchanged 
during discussions. In Dakar, the emergence of a mixture of Wolof and French was observed whereas, in Ziguinchor, the mixing of languages was a sign of the user's plural identifications and of the maintenance of an ethnic identity. Mixed speech is characterized by mainly extraphrastic alternations and by borrowings in the base language, which itself may also alternate between the language of one's group or wolof.

Mots-clés/keywords : Sénégal, Wolof, alternance codique ou code switching, français, jeunes, villes/Senegal, Wolof, code switching, French, children, cities. 